\title{
Variable-Length Particle Swarm Optimisation for Feature Selection on High-Dimensional Classification
}

\author{
Binh Tran, Member, IEEE, Bing Xue, Member, IEEE, and Mengjie Zhang, Senior Member, IEEE
}

\begin{abstract}
With a global search mechanism, Particle Swarm Optimisation (PSO) has shown promise in feature selection. However, most of the current PSO-based feature selection methods use a fix-length representation, which is inflexible and limits the performance of PSO for feature selection. When applying these methods to high-dimensional data, it not only consumes a significant amount of memory but also requires a high computational cost. Overcoming this limitation enables PSO to work on data with much higher dimensionality which has become more and more popular with the advance of data collection technologies. In this study, we propose the first variable-length PSO representation for feature selection, enabling particles to have different and shorter lengths, which defines smaller search space and therefore, improves the performance of PSO. By rearranging features in a descending order of their relevance, we facilitate particles with shorter lengths to achieve better classification performance. Furthermore, using the proposed length changing mechanism, PSO can jump out of local optima, further narrow the search space and focus its search on smaller and more fruitful area. These strategies enable PSO to reach better solutions in a shorter time. Results on ten high-dimensional datasets with varying difficulties show that the proposed variable-length PSO can achieve much smaller feature subsets with significantly higher classification performance in much shorter time than the fixedlength PSO methods. The proposed method also outperformed the compared non-PSO feature selection methods in most cases.
\end{abstract}

Index Terms-Classification, data mining, feature selection, particle swarm optimisation, high-dimensional data.

\section{INTRODUCTION}

Recently, feature selection (FS) has become an essential technique in data preprocessing especially on highdimensional data. With the tremendous growth in data collection technologies, the number of features collected in many machine learning applications becomes increasingly larger. However, the existence of irrelevant and redundant features in these datasets may obscure the relevant ones, which significantly degrades the performance of many learning algorithms. Therefore, with the aim of eliminating irrelevant and redundant features, FS helps in improving the accuracy and interpretability of the learnt models, shortening the learning time, and reducing the storage space of the dataset [1].

Researchers have proposed a large number of FS methods for classification problems, which can be classified into wrapper and filter approaches [2]. While a wrapper method

B. Tran, B. Xue, and M. Zhang are with the School of Engineering and Computer Science, Victoria University of Wellington, Wellington 6140, New Zealand (e-mail: binh.tran@ecs.vuw.ac.nz; bing.xue@ecs.vuw.ac.nz; mengjie.zhang@ecs.vuw.ac.nz) evaluates the goodness of a feature subset using a classification algorithm, a filter method is based solely on the intrinsic characteristic of the training data. Therefore, wrappers can usually obtain better classification performance than filters, but with higher computation time. Filter methods are also said to be more general than wrappers. Therefore, a combination of these two approaches has also been proposed to combine their strengths [3].

Although being studied for decades, FS is still a challenging task especially on high-dimensional data due to its huge search space. FS is a combinatorial optimisation problem with $2^{n}$ possible combinations, where $n$ is the number of original features. In other words, the search space grows exponentially with the number of features.

By ranking features individually, feature ranking or feature weighting methods [4] usually scale well with highdimensional data. Features are ranked based on their degrees of relevance to the target concept. Then a predefined number of top-ranked features will be selected to form the final subset. However, it is difficult to determine an appropriate number of features to select without a certain amount of domain knowledge or extensive trials. Furthermore, there can be two-way, three-way or multi-way complex interactions among features [5]. An individually weakly relevant feature may become highly useful when combined with other features and vice versa. By evaluating features independently, these methods can not handle feature interactions. In addition, the top-ranked features may be redundant, which may degrade the performance of classification algorithms. An improvement of feature ranking approaches is to append a second stage where a heuristic search is applied to the top-ranked features to remove less relevant and redundant features [6]. However, since features are individually ranked in the first stage, this approach may fail to identify multi-way feature interactions.

In contrast with feature ranking, feature subset selection methods can evaluate the whole feature subset at once, which can better deal with feature interactions. Sequential forward selection (SFS) [7] and backward FS methods (SBS) [8] are typical feature subset selection methods. SFS (SBS) gradually adds (removes) features until no further improvement. While SFS can be efficient in high-dimensional data, SBS is too expensive to apply to these datasets [3]. However, using a greedy search, SFS and similar methods are prone to be stuck in local optima, especially in a search space with thousands of features. A global search technique is needed to explore this huge search space better. 
Particle Swarm Optimisation (PSO) proposed by Kennedy and Eberhart [9] is a population-based algorithm which is well-known with global search ability. Simulating the social behaviour of bird flocking, PSO works by maintaining a swarm of particles, each of which represents a candidate solution. By communicating their best found solutions, these particles can fly towards more fruitful areas and discover better solutions. PSO has been applied and shown promise in many problems [10], [11]. However, most of its applications are usually on low dimensionality with tens or hundreds of features [12], [13]. Its performance on high-dimensional data with thousands of features or more is still limited due to the following limitations which motivate us to propose a new PSO-based FS method.

\section{A. Motivations}

First of all, most of the PSO-based FS methods in the literature use the fix-length representation. In other words, all the particles in the population have the length which is equal to the original number of features. With this representation, PSO usually requires a significant amount of memory and computation time when applying to FS on high-dimensional data. This limitation hinders PSO's applications on problems with hundreds of thousands of features, which become more popular in recent years. Furthermore, representation is the main factor in defining the size of the search space. An effective and flexible representation can help PSO achieve better solutions. In this study, we propose the first PSO-based FS algorithm with a variable-length representation, so called VLPSO.

Different particles can have different lengths (i.e. numbers of features). Therefore, they may focus on different areas of the search space. Based on this ability, we propose a new initialisation method called population division, which divides the population into divisions of particles with different lengths to provide an appropriate level of diversity for the whole population.

Furthermore, to encourage the short-length particles to find good feature subsets, we rearrange features in the descending order of their importance or relevance to the target concept. In other words, features are ranked based on a feature ranking method before applying PSO. In this way, the most important features can always be selected by any particle in the swarm. On the other hand, particles with longer lengths will have the potential to include less relevant features, enabling PSO to detect possible feature interactions that can lead to better feature subsets.

To facilitate particles with different lengths to learn from each other, VLPSO adopts the updating mechanism proposed in the comprehensive learning PSO (CLPSO) [14], which is a PSO variant. By allowing any particle to become an exemplar for others to learn from, CLPSO encourages diversity of the swarm and eliminates the need for specifying a specific communication topology. Furthermore, different dimensions of a particle can also learn from different particles. These characteristics of CLPSO enable our variable-length particles to choose appropriate exemplars easier. CLPSO has achieved significantly better results than many other PSO variants on many complex multimodal functions [14], [15]. However, to the best of our knowledge, CLPSO has never been applied to FS. In this study, we will apply CLPSO to FS with some adjustments. Firstly, the exemplar assignment in CLPSO needs to be adjusted to suit the newly proposed representation. Additionally, the probability used to choose exemplars in CLPSO is based on the index of the particle, which may limit its performance. Therefore, VLPSO will use an adaptive learning probability recently proposed in [15] to overcome this limitation.

Furthermore, we propose a length changing mechanism to alleviate the premature convergence, which is a common problem of PSO, especially on high-dimensional data. This mechanism enables particles to change length during the evolution. It helps PSO escape from local optima and move to more fruitful areas in the search space.

Additionally, PSO is well-known with the capability of quickly detecting fruitful regions; however, once there, it may not perform a refined local search well in complex search space to compute the optimum with high accuracy [16]. Local search has been combined with PSO to overcome this drawback [3]. Therefore, we also combine VLPSO with local search to further improve VLPSO performance on highdimensional data.

Besides search mechanism, feature evaluation is another critical component of a FS method.

Although wrapper methods usually obtain better classification performance than filters, using classification accuracy solely may not be sufficiently powerful to distinguish the subtle difference between feature subsets. When working on highdimensional data, classification algorithms require a much larger number of instances to maintain their performance due to the curse of dimensionality, which is usually not satisfied in reality. Therefore, we combine the strengths of both wrapper and filter approaches aiming to provide a smoother fitness landscape to facilitate the search process.

To avoid adding a notable amount of computation, we use a hybrid evaluation method of K-nearest neighbour (KNN) and a distance measure proposed in [3]. Since both use on the same distance measure in their calculations, the increase in evaluation time is neglectable.

\section{B. Goals}

The main goal of this paper is to propose the first variablelength representation in PSO for effective and efficient FS. Specifically, we will investigate the following research objectives:

- How to design particles with different lengths that can communicate smoothly with each other;

- Whether the feature subsets selected by the proposed algorithm are smaller and achieve similar or better classification performance than the original feature sets, and the subsets selected by standard PSO and other compared PSO-based FS methods;

- Whether incorporating a local search procedure helps the proposed method achieve even higher classification accuracy; 
- Whether the proposed methods significantly reduce the running time of PSO on high-dimensional data;

- Whether the proposed methods outperform traditional FS methods; and

- How effective and efficient the proposed strategies help PSO improve its performance on high-dimensional data.

\section{BACKGROUND AND RELATED WORK}

\section{A. Particle Swarm Optimisation}

As a population based algorithm, PSO [9] maintains a swarm of particles. Each particle has a position which represents a candidate solution and a velocity showing the speed and direction that the particle should move in the next iteration. A particle's position and velocity are encoded in two vectors of $n$ real numbers where $n$ is the dimensionality of the problem. Particle's position is evaluated based on a fitness function. Then the best position that each particle has explored so far (pbest) is recorded and shared with other particles. In the conventional PSO, a fully connected topology is used to find the best position of the whole population (gbest). In other topologies which do not connect all particles, gbest is replaced with a local best (lbest). These best positions are used to update a particle's velocity which then defines its position as shown in Eqs. (1) and (2).

$$
\begin{gathered}
v_{i d}^{t+1}=w * v_{i d}^{t}+c_{1} * r_{1 i} *\left(p_{i d}^{t}-x_{i d}^{t}\right)+c_{2} * r_{2 i} *\left(p_{g d}^{t}-x_{i d}^{t}\right) \\
x_{i d}^{t+1}=x_{i d}^{t}+v_{i d}^{t+1}
\end{gathered}
$$

where $v_{i d}^{t}$ and $x_{i d}^{t}$ are the velocity and position of the $i^{t h}$ particle in dimension $d$ at time $t . w$ is the inertia weight representing the moving momentum of the particles. $p_{i d}^{t}$ and $p_{g d}^{t}$ are pbest and gbest positions in dimension $d$ at time $t . c_{1}$ and $c_{2}$ are acceleration constants, and $r_{1}$ and $r_{2}$ are random values uniformly distributed in $[0,1]$.

When applying PSO to FS, each real value ranging from 0 to 1 in the position vector indicates whether the corresponding feature should be selected or not based on a predefined threshold (e.g. 0.6).

\section{B. Comprehensive Learning PSO}

Comprehensive Learning PSO (CLPSO) proposed by Liang et al. in [14] is a variant of continuous PSO in which a particle can learn from pbest of any particle. This strategy helps PSO maintain the diversity of the swarm and hence alleviate the common problem of premature convergence in PSO. Furthermore, while in standard PSO, all dimensions of a particle's velocity are updated based on its pbest and gbest, CLPSO enables different dimensions to learn from pbest of different particles including its own. The decision of choosing itself or another particle as an exemplar depends on a learning probability called $P c$ ranging from 0.05 to 0.5 . Each particle has its own $P c$. Eq. (3) is used to calculate $P c$ for the $i^{t h}$ particle.

$$
P c_{i}=0.05+0.45 \frac{\exp ^{\frac{10(i-1)}{S-1}}}{e x p^{10}-1}
$$

where $S$ is the population size.
To set an exemplar for a dimension $d$ of the $i^{\text {th }}$ particle, CLPSO generates a random number. If this number is greater than $P c_{i}, d$ learns from its own pbest; otherwise, a tournament selection with the size of 2 will be used to choose the exemplar for $d$. Therefore, besides a position and a velocity vector, CLSPO has another vector to record exemplars, which are the indexes of the chosen particles, for all dimensions. Exemplars of a particle remain unchanged until it stops improving for $\alpha$ iterations. Therefore each particle also counts how many iterations that its pbest has not been changed. When this count exceeds $\alpha$, all exemplars of this particle are renewed. With these changes, CLPSO uses Eq. (4) to update velocity.

$$
v_{i d}^{t+1}=w * v_{i d}^{t}+c * r_{i d} *\left(p_{\text {exmplr }(i d) d}^{t}-x_{i d}^{t}\right)
$$

where exmplr $(i d)$ returns the exemplar of particle $i$ in dimension $d$.

\section{PSO for FS in Classification on High-Dimensional Data}

PSO has been proposed and shown promise in FS [17]. An increased interest in PSO has shown through a growing number of papers proposing PSO-based FS methods in the past ten years [12].

Both filter or wrapper approaches have been proposed in PSO-based FS methods. In filter methods, different measures were proposed to evaluate feature subsets such as rough set [18], fuzzy consistency [19], mutual information, and entropy [20]. On the other hand, feature evaluation methods in wrappers are based on the classification performance of a learning method [17]. Combination of both approaches has also been proposed [3].

To improve PSO performance for FS, researchers have also proposed many improvements in updating mechanisms of gbest [17], pbest [3] and particles [21], [22], communication topology [23], initialisation [17], and representation [24]. Readers are referred to [12] for more comprehensive survey. In this section, we only focus on reviewing those methods that improve PSO representation.

Reducing PSO search space by explicitly eliminating redundant features is an effective way to improve PSO performance. In [24], Lane et al. used a statistical clustering method to group similar features into the same cluster. Then, during the evolutionary process, some features with the highest probability (i.e. velocity) from each cluster were selected. Results showed that these methods could select a smaller number of features to achieve similar or better classification performance than all features and the compared methods. However, it is not easy to choose an appropriate number of features that should be selected from each cluster.

Among the early PSO variants, binary PSO [25] seems to significantly reduce the search space over continuous PSO (and also the memory space) when it restricts the position vector into binary values. However, using velocity solely to update its position, binary PSO cannot achieve a good performance [26]. Different updating mechanisms have been proposed to improve its performance [22], [26]. Nevertheless, these methods still use a fixed-length representation, which cannot scale well 
when the number of features reaches hundreds of thousands or even millions.

To provide a better solution for discrete optimisation problems, Chen et al. [27] proposed a set-based PSO method (SPSO) in which a particle is encoded as a crisp set of elements. Velocity is a set of elements and their corresponding possibilities. Results on the travelling salesman and multidimensional knapsack problems showed that S-PSO and its variants [28] were promising in solving discrete problems. However, the position and velocity representations in S-PSO require even more memory than standard PSO.

Another set-based representation (named SBPSO) was proposed by Langeveld et al. [29] where the position is a set of elements while velocity is a set of operation pairs representing adding or removing elements. To avoid early convergence, velocity is updated using not only the pbest and lbest sets but also two more sets generated by two proposed operators. One is a random removal of some elements that appear in current position, pbest and lbest sets. Another operator is adding some elements that are not in these three sets using a ktournament selection that involves further fitness evaluations, which may lead to much higher computation time if the fitness function is costly. Results on knapsack problems showed that SBPSO performed significantly better than other three discrete PSO algorithms. However, the sensitivity analysis of SBPSO showed that its performance is sensitive to parameters used in the velocity formula [29].

In summary, although many PSO-based FS methods have been proposed to improve PSO performance in FS, not many studies addressed the limitation of the PSO representation in solving FS [12]. A new representation that can improve the scalability of PSO for FS, especially on high-dimensional data, is still missing. In the following section, we will propose a new approach to tackling the limitation of the fixed-length representation in PSO for FS.

\section{The Proposed Method}

This section starts with a description of the proposed variable-length representation along with the exemplar assignment and an adaptive learning probability that are adjusted based on CLPSO. It then introduces the population division, feature ranking and length changing strategies that are enabled by the variable-length representation. Finally, it presents the hybrid fitness function, the overall algorithm of VLPSO and the local search that is combined with VLPSO.

\section{A. Variable-Length PSO Representation}

The proposed variable-length representation aims to improve the scalability of PSO for FS on higher dimensional problems and reduce the computation time required when using a fix-length PSO method for FS.

The proposed representation is still vector-based as the traditional PSO; however, each particle will have a different length $L$. VLPSO is developed based on the CLPSO [14] which was described in Section II-B. Fig. 1 shows the representation of a VLPSO particle with length $L$, which has three vectors including the position, velocity and exemplar. Two additional

\begin{tabular}{|c|c|c|c|c|c|c|c|c|c|c|}
\hline Dimension: & 1 & 2 & 3 & 4 & 5 & 1 & 2 & 3 & $\ldots$ & $L$ \\
\hline Position: & 0.8 & 0.3 & 0.9 & 0.4 & 0.1 & 0.6 & 0.2 & 0.7 & $\ldots$ & 0.5 \\
\hline Velocity: & 0.1 & 0.2 & 0.5 & 0.4 & 0.1 & 0.2 & 0.4 & 0.3 & $\ldots$ & 0.2 \\
\hline Exemplar: & 8 & 7 & 5 & 6 & 2 & 3 & 7 & 8 & $\ldots$ & 1 \\
\hline \multicolumn{6}{|c|}{ Learning Probability $(\mathrm{Pc})=0.25$} & \multicolumn{5}{|c|}{ Renew Exemplar Count $=$} \\
\hline
\end{tabular}

Fig. 1. Representation of a VLPSO particle with length $L$

variables record its learning probability $(P c)$ and the renew exemplar count (i.e. the number of iterations that pbest has not been improved).

The velocity and position updating in VLPSO follow Eqs. (4) and (2), respectively.

\section{B. Exemplar Assignment}

In the original CLPSO, any particle can be used as an exemplar for a dimension of any particle. However, since different VLPSO particles have different lengths, the exemplar chosen for a particular dimension needs to have the corresponding dimension. In other words, the exemplar's length must exceed that dimension. Therefore, we propose a new exemplar updating mechanism for VLPSO, whose pseudocode is shown in Algorithm 1. The main difference between this method with the original one in CLPSO is that the two exemplars $\left(p_{1}\right.$ and $\left.p_{2}\right)$ are randomly sampled (Lines 9-10) until both of them satisfy the above mentioned condition. However, if this condition is not met after a number of attempts, its own pbest will be used as the exemplar. In this method, the number of attempts is simply set to the population size.

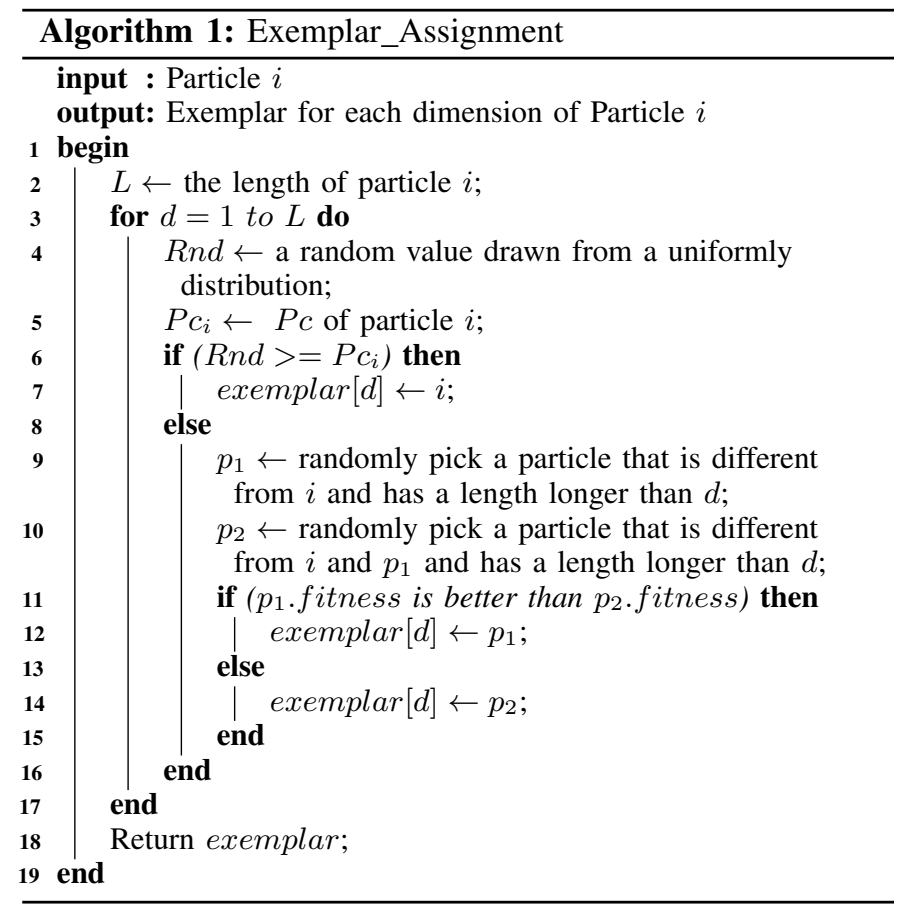




\section{Adaptive Learning Probability}

In the original CLPSO, the probability of choosing exemplars for each dimension of a particle $(\mathrm{Pc})$ is set based on its identity or index in the population and kept unchanged during the evolutionary process. As can be seen from Eq. (3), particles with a smaller index will have smaller $P c$ than those with a greater index. Therefore, according to the use of $P c$ for choosing exemplars in CLPSO, it is more likely that the smallindex particles will follow its own pbest. However, to locate a better position or solution, particles should learn from particles with better fitness. Therefore, only those particles with better fitness should have smaller $P c$, so that they can continue to exploit their good direction to find better pbest. In contrast, the worse particles should learn from the better ones. With this rationale, Yu et al. [15] proposed to calculate $P c$ for a particle based on its fitness rank instead of its index. As shown in Eq. (5), the proposed strategy assigns a smaller $P c$ for a particle with a smaller rank (i.e. a fitter particle). In this study, we adopt this strategy since it has shown promise in improving the performance of CLPSO for function optimisation [15].

$$
P c_{i}=0.05+0.45 \frac{\exp ^{\frac{10(\operatorname{rank}(i)-1)}{S-1}}}{\exp ^{10}-1}
$$

where $S$ is the population size and $\operatorname{rank}(i)$ is the rank of particle $i$. The best particle in the swarm will be ranked 1 .

\section{Population Division}

Based on the variable-length representation, VLPSO enables particles in the population to have any length that is smaller than the dimensionality of the problem. However, this may degrade PSO performance since particles will not learn much from each other when they are too different. Therefore, instead of setting a different length for each particle, we divide the whole population into a predefined number of divisions. In this way, we divide the search space into smaller subspaces, which can improve PSO's performance, especially in such a large and complex search space as in high-dimensional data.

The number of particles (or size) of each division (DivSize) is calculated based on the population size (PopSize) and the number of divisions ( $N b r D i v)$ as shown in Eq. (6). Particles in the same division will have the same length. The length of particles in a division $V\left(\operatorname{ParLen}_{V}\right)$ is calculated based on Eq. (7), where the maximum length (MaxLen) is the dimensionality of the problem. Note that within a division, particles with the same length can represent different feature subsets with different feature subset sizes. For example, two particles of length 8 represent two solutions, 10100001 and 10001111, which show which features are selected from the first 8 features of the given dataset. They are corresponding to two feature subsets, $\{F 1, F 3, F 8\}$ and $\{F 1, F 5, F 6, F 7, F 8\}$ which have the feature subset size of 3 and 5 , respectively. Note that the $d^{t h}$ dimension always represents the $d^{\text {th }}$ feature in all particles. This enables particles to learn from each other despite of their different lengths.

$$
\text { DivSize }=\frac{\text { PopSize }}{\text { NbrDiv }}
$$

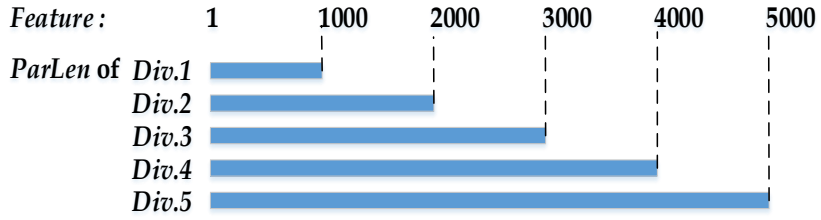

Fig. 2. An example of population division for a problem with 5000 features and the number of division is 5 .

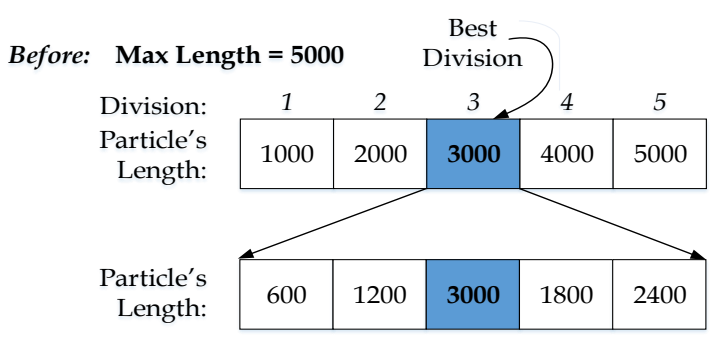

After: $\quad$ Max Length $=3000$

Fig. 3. An example of length changing in a swarm with 5 divisions.

$$
\operatorname{ParLen}_{V}=\operatorname{MaxLen} * \frac{V}{N b r D i v}
$$

Fig. 2 shows an example of the particle lengths in a problem with 5000 features and the number of divisions is set to 5 . The length of all particles in Division 1 will be 1000 . They will search for good feature subsets in the first 1000 features. Similarly, Division 2 will focus on feature subsets in the first 2000 features.

\section{E. Feature Ranking}

To rearrange features in the descending order of their relevance, we can use any measure to evaluate features. In this study, we use the symmetric uncertainty (SU) [30] since it is a non-parametric measure and commonly used in FS methods [6], [31]. SU is a normalised version of information gain (IG) to evaluate feature relevance. To rank features, we use SU as shown in Eq. (8) to measure the correlation between a feature $F$ and the class label $C$. The higher a feature correlates to the class label, the better it is.

$$
\begin{aligned}
S U(F, C) & =\left[\frac{I G(F \mid C)}{H(F)+H(C)}\right] \\
I G(F \mid C) & =H(F)-H(F \mid C)
\end{aligned}
$$

where $H(F)$ is the entropy of $F$ and $H(F \mid C)$ is the conditional entropy of $F$ given $C$. The value of $S U(F, C)$ ranges from 0 to 1 , where 1 represents the most relevant feature.

\section{F. Length Changing}

During the evolutionary process, to help PSO jump out of possible local optima, we propose a length changing mechanism to direct particles to more fruitful areas in the search space, enabling PSO to reach better solutions in a shorter time. Particularly, when gbest does not change for a predefined 
number of iterations, we calculate the average fitness of all particles in each division and resize the particles to scale PSO search into the best division. In other words, the particle length of the best division will become the maximum length of the swarm after length changing. During this process, we keep the particles in the best division unchanged and resize those in the other divisions to a shorter length than the new maximum length. The process automatically changes the particles' length by cutting or appending more dimensions at the end of the representation while keeping the learnt knowledge in the other dimensions. The number of dimensions being cut or appended is dynamically calculated based on the new length and the current length.

Fig. 3 shows a demonstration of this process on a swarm with five divisions. Initially, particle length of division 1, 2, 3,4 and 5 are 1000, 2000, 3000, 4000, and 5000, respectively. Suppose the third division is be the best division with the highest average fitness, it is kept unchanged and 3000 becomes the new maximum length of the swarm. However, particle length of division $1,2,4$ and 5 will be changed to $600,1200,1800$, and 2400 , respectively. Therefore, the last 400, 800, 2200 and 2600 dimensions will be cut in particle representations of division 1,2, 4 and 5, respectively.

Algorithm 2 shows the pseudocode of the length changing procedure. If the current length of particles in a division is shorter than the new length, then more dimensions will be appended and randomly initialised (Lines 12-16); otherwise, the exceeding dimensions will be removed (Lines 18-21).

Since the maximal length of particles is always getting shorter every time particles' length is changed, another benefit of this mechanism is a dramatically reduction in the PSO computation time.

This length changing is applied when gbest does not improved for a number of iterations $(\beta)$. $\beta$ should be large enough for PSO to converge, and small enough for PSO to avoid being stuck in local optima for too long. Therefore, we conducted a sensitivity analysis as described in Section IV-D to choose suitable values for $\beta$ and the number of divisions.

\section{G. Fitness Function}

To combine the strengths of the wrapper and filter methods without significantly increasing the computation time, VLPSO uses the fitness function proposed in [3] to combine the accuracy of K-nearest neighbour (KNN) and a distance measure [32] using a weight $(\gamma)$ as shown in Eq. (10). While the classification accuracy can measure the performance of the feature subset, the distance measure can approximate how far these features can separate instances of different classes and unite those of the same class.

$$
\text { fitness }=(\gamma \cdot \text { accuracy }+(1-\gamma) \cdot \text { distance })
$$

To deal with unbalanced data in many high-dimensional datasets, we used a balanced accuracy [33] calculated based on Eq. (11) for the first component in the fitness function. Leave-one-out cross validation on the training data is used to evaluate the performance of KNN.

$$
\text { balanced_accuracy }=\frac{1}{c} \sum_{i=1}^{c} T P R_{i}
$$

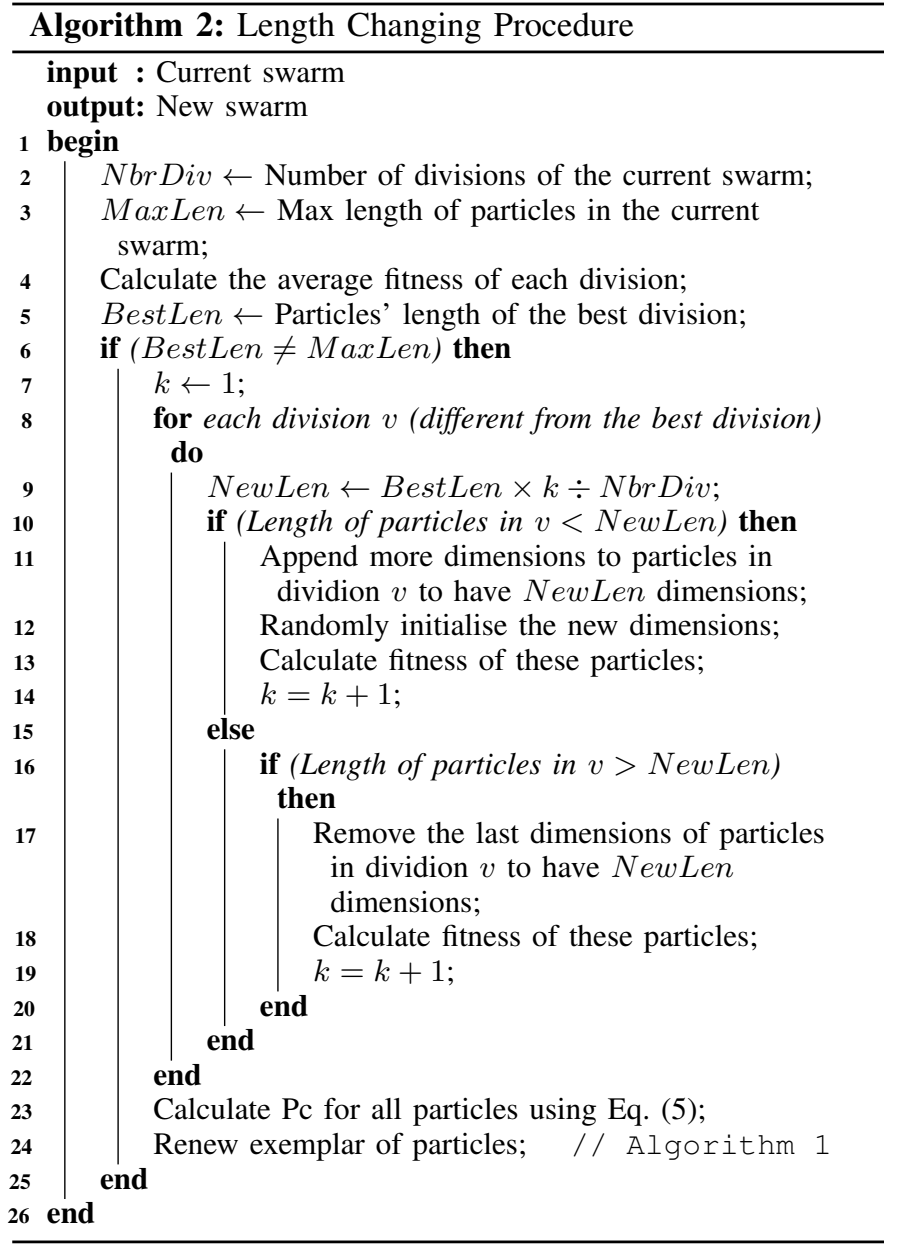

where $c$ is the number of classes of the problem, and $T P R_{i}$ is the true positive ratio or the proportion of correctly identified instances in class $i$. Since there is no bias to any specific class, the weight for each class is set to $1 / c$.

The distance measure is calculated based on Eq. (12), which aims at maximising the distance between instances of different classes $\left(D_{b}\right)$ and minimising the distance between instances of the same class $\left(D_{w}\right)$.

$$
\text { distance }=\frac{1}{1+\exp ^{-5\left(D_{b}-D_{w}\right)}}
$$

where

$$
\begin{aligned}
& D_{b}=\frac{1}{M} \sum_{i=1}^{M} \min _{\left\{j \mid j \neq i, \operatorname{class}\left(I_{i}\right) \neq \operatorname{class}\left(I_{j}\right)\right\}} \operatorname{Dis}\left(I_{i}, I_{j}\right) \\
& D_{w}=\frac{1}{M} \sum_{i=1}^{M} \max _{\left\{j \mid j \neq i, \operatorname{class}\left(I_{i}\right)=\operatorname{class}\left(I_{j}\right)\right\}} \operatorname{Dis}\left(I_{i}, I_{j}\right)
\end{aligned}
$$

where $M$ is the number of instances in the training set. The distance between two instances $\operatorname{Dis}\left(I_{i}, I_{j}\right)$ can be measured based on any distance approximation methods. In this study, we use Manhattan measure since it is preferable than Euclidean distance metric for high-dimensional data [34]. To appropriately apply this distance measure, the training data is scaled to the range of $[0,1]$. 


\section{H. VLPSO Overall Algorithm}

Fig. 4 shows the flowchart of VLPSO algorithm. It has three inputs, the number of divisions ( $N b r D i v)$, the maximum iterations that pbest is not improved to renew exemplars $(\alpha)$ for a particle, and the maximum iterations that gbest is not changed $(\beta)$ to change particles' lengths. VLPSO starts with rearranging features based on the feature ranking described in Section III-E. After that, it initialises all divisions in the first loop, then calculates the learning probability $P c$ and assigns exemplars for each particle. The second loop is the evolutionary process. It repeats until reaching the maximum number of iterations. During this evolution, if gbest is not improved for $\beta$ times, length changing procedure is called. $P c$ is also adapted based on Eq. (5).

The computation time of the proposed method and the baseline methods can be divided into two parts, time for PSO updating and time for fitness evaluation, in which fitness evaluation usually accounts for a much larger portion between the two. The former can be calculated based on the number of iterations $(I)$, the number of particles $(P)$, and the length of the particles which is equal to the number of original features $(N)$ for PSO and ECLPSO. By dividing the population into $D$ divisions, each of which has $P / D$ particles with the particle length of $1 * N / D, 2 * N / D, \ldots$, or $D * N / D=N$, the proposed method reduces the time for PSO updating by half. For the fitness evaluation, the computation time highly depends on the number of features selected by each particle, which is hard to estimate and dataset-dependent. However, in the worse case that all particles select all features, the proposed method still takes only half of the time required by the baseline methods due to its shorter particle lengths.

\section{VLPSO with Local Search}

To further improve the performance of VLPSO on high dimensional classification problems, we apply local search to pbest which was proposed in [3]. We call the combined method of VLPSO with local search as VLPSO-LS. This local search process aims to find a better solution surrounding the newly found pbest by randomly removing some redundant features and adding more relevant ones. The SU measure based on Eq. (8) is also used to evaluate feature relevance and redundancy in this process.

Given a binary vector corresponding to the feature subset of pbest, the local search procedure conducts a given number of tries. The more local search tries, the better solution can be found. Therefore, we set the number of tries to 100, which is equal to the maximum number of iterations PSO runs. However, thanks to the fast fitness evaluation used in the local search, 100 evaluations will not cost as much as in PSO. Each local search try considers to flip a random portion of pbest based on a given flipping size to create a new pbest. The size of the random portion is dynamically determined and proportional to the current pbest size. $25 \%$ is chosen to encourage removing more redundant features and adding more relevant ones in one local search try. The flipping process will scan features in this random portion to remove selected features if they are redundant and add non-selected features

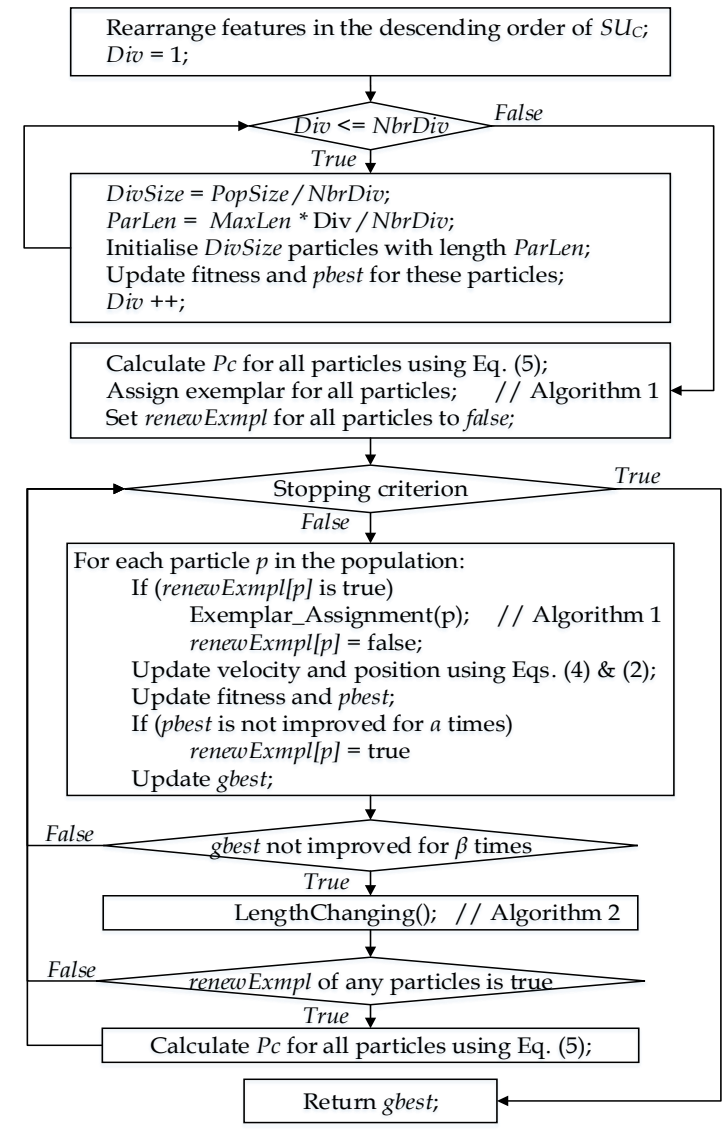

Fig. 4. Overall variable-length PSO algorithm.

if they are relevant. A feature is defined as redundant if it is more correlated to other selected features than to the class label. A feature is relevant if it is more correlated to the class than the average correlation of all the selected features in the random portion. Therefore, the number of features actually flipped is not only dynamically determined by the current pbest size but also the characteristic of the dataset. As a result, the performance of the local search is not highly sensitive to the values of these two related parameters.

If a better pbest is found, it will replace the current one. Each local search try involves an evaluation process. Therefore, a significant number of evaluations will be added, which substantially increases the computation time. Therefore, to speed up VLPSO-LS, we also use the fast fitness evaluation strategy [3], which calculates the new distance between instances by adding to or subtracting from the current distance the value difference in features that are added or removed from the current pbest, respectively. Since a small portion of pbest is flipped in one local search try, this strategy saves a significant amount of computation, leading to much shorter evaluation time.

The frequency of applying local search can be predefined to compromise between its effectiveness and efficiency. When the trigger condition is satisfied, VLPSO-LS applies the local search procedure to the newly found pbest. 
TABLE I

DATASETS

\begin{tabular}{|l|r|r|r|r|r|}
\hline Dataset & \#Features & \#Ins. & \#Class & $\begin{array}{r}\text { \%Smallest } \\
\text { class }\end{array}$ & $\begin{array}{r}\text { \%Largest } \\
\text { class }\end{array}$ \\
\hline SRBCT & 2,308 & 83 & 4 & 13 & 35 \\
\hline Leukemia 1 & 5,327 & 72 & 3 & 13 & 53 \\
\hline DLBCL & 5,469 & 77 & 2 & 25 & 75 \\
\hline 9Tumor & 5,726 & 60 & 9 & 3 & 15 \\
\hline Brain Tumor 1 & 5,920 & 90 & 5 & 4 & 67 \\
\hline Brain Tumor 2 & 10,367 & 50 & 4 & 14 & 30 \\
\hline Prostate & 10,509 & 102 & 2 & 49 & 51 \\
\hline Leukemia 2 & 11,225 & 72 & 3 & 28 & 39 \\
\hline 11Tumor & 12,533 & 174 & 11 & 4 & 16 \\
\hline Lung Cancer & 12,600 & 203 & 5 & 3 & 68 \\
\hline
\end{tabular}

\section{EXPERIMENT DESIGN}

\section{A. Datasets}

We used ten gene expression datasets with thousands of features that are publicly available on http://www.gemssystem.org. Table I shows the number of features, instances, classes, and the percentage of instances in the smallest and largest class of each dataset. As can be seen from Table I, these datasets have a much smaller number of instances compared to the number of features. They are also highly unbalanced data. These characteristics make them become very challenging problems for both FS and classification.

\section{B. Experiment Setting}

Due to the small number of instances in these datasets, 10fold cross validation is used to create training and test sets for the experiments (no validation set involved). One fold is kept as unseen test data, never used during the FS process. The remaining 9 folds form the training data. Only the training data is used to during the FS process. After FS is finished, the training and test sets will be transformed based on the selected features and put into KNN to evaluate the performance of the FS method.

\section{Baseline Methods}

To evaluate the performance of VLPSO and VLPSO-LS, we compared the classification accuracy of KNN using the features selected by both methods with the original full feature sets, and the feature subsets selected by standard PSO (or PSO for short). They are also compared with the comprehensive learning PSO enhanced with the adaptive learning probability [15] described in Section III-C, which we call ECLPSO for presentation convenience. All the compared methods will use the same fitness function and settings for common parameters. We also compare our methods with a recently proposed PSObased feature selection method for high-dimensional classification using a competitive swarm optimizer (CSO) [21]. In this method, all particles are divided into two groups where pairwise comparison are applied and the better particle between the two will be used as an exemplar for the other. $\mathrm{KNN}$ is also used to evaluate feature subsets. We run the code provided by the authors on the same settings as other compared methods.
TABLE II

PARAMETER SETTINGS

\begin{tabular}{|l|l|}
\hline Parameters & Settings \\
\hline Population Size & \#features/20 (restriction to 300) \\
Maximum iterations & 100 \\
$c 1=c 2$ or $c$ & 1.49445 \\
$w$ & $0.9-0.5 * \frac{\text { current iteration }}{\text { max iteration }}$ \\
Threshold for selected feature & 0.6 \\
\hline Communication topology & Fully connected (PSO) \\
\hline Max iterations for renew exem- & 7 (ECLPSO, VLPSO, VLPSO- \\
plars $(\alpha)$ & LS) \\
\hline Local search tries & 100 (VLPSO-LS) \\
Local search flipping size & $25 \%$ of current pbest's size \\
& (VLPSO-LS) \\
\hline Number of divisions & 12 (VLPSO, VLPSO-LS) \\
Max iterations for length chang- & 9 (VLPSO, VLPSO-LS) \\
ing $(\beta)$ & \\
\hline
\end{tabular}

We also compared VLPSO-LS with three traditional FS methods, which are the linear forward selection (LFS), the correlation-based FS (CFS) [35], and the fast correlationbased FS method (FCBF) [6]. We chose these feature subset selection methods because of their popularity and the ability to automatically determine the number of selected features as our proposed methods. LFS is derived from the sequential forward selection (SFS) where features are gradually added until no further improvement in classification accuracy. By restricting the number of features to be considered in each step, LFS [36] runs faster and finds smaller feature subsets with better classification performance than SFS. While LFS uses a wrapper approach, CFS is a filter FS method using the correlation measure to bias towards feature subset containing more relevant features and less redundant ones. Since bestfirst search is too expensive, especially on high-dimensional data, we ran CFS with a greedy forward selection. Unlike LFS and CFS, FCBF is a two-stage FS method where features are first ranked using the correlation measure and sorted in the descending order of relevance. Then a heuristic search is used to scan the ordered list to remove redundant features. Weka [37] was used to run the three methods with their default settings.

\section{Parameter Settings}

Table II shows the parameter settings used in the experiments. As can be seen from Table I, the datasets have very different numbers of features ranging from 2,000 to 12,000. To deal with the large difference in the search space of different datasets, we set the population size to one twentieth of the number of features, but limited to 300 due to limited memory for computation. The parameter settings for the local search in VLPSO-LS are the same as in [3]. The maximum iterations for renewing exemplars $(\alpha)$ is set to 7 as suggested in [14]. The threshold for selected feature is usually set to 0.5 or slightly larger [17], [38]. Within a reasonable range, e.g. [0.5, 0.7], the value of this parameter does not significantly influence the selection process as investigated in [38]. The reason is during the evolutionary process PSO can automatically update the 


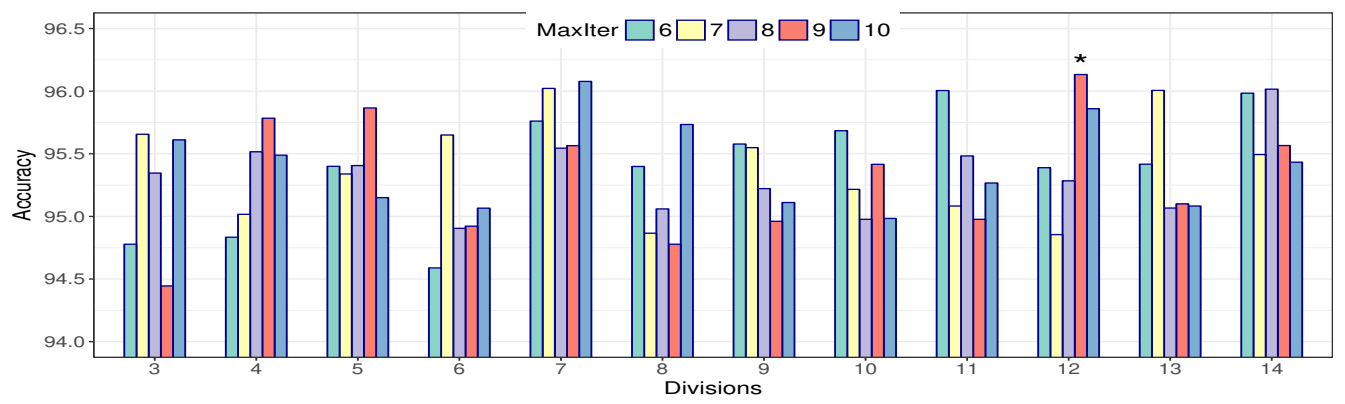

Fig. 5. Average results of 60 combinations of two parameters (number of divisions and maximum iterations to change particles' lengths).

particles' position values to make a feature become selected or not. PSO position updating is guided by fittest particles with better feature subsets. Therefore, PSO can adjust particle position values to make them higher or lower than the given threshold so that the feature subsets can obtain better fitness. We chose 0.6 to slightly prefer a smaller number of features at the early stage of the evolution.

The numbers of divisions and maximum iterations that gbest stays unchanged before changing particles' lengths $(\beta)$ are new parameters that were proposed for variable-length PSO. Therefore, we conducted an experiment (sensitivity analysis) to find the appropriate values of these two parameters. The DLBCL dataset was used in this experiment because it has a medium size compared with other datasets. VLPSO-LS was run with 12 different values for the number of divisions ranging from 3 to 14 , and 5 values from 6 to 10 for the maximum iterations to change particles' lengths, resulting in 60 combinations of these two parameters. Each combination was run 30 times. Fig. 5 shows the average test results of each combination with the best accuracy marked with an asterisk (*). The best combination was $\{12$ divisions, 9 iterations $\}$ which VLPSO and VLPSO-LS used for all datasets.

Since PSO is a stochastic algorithm, 30 independent runs of each method with 30 different seeds are executed on each training set. As a result, each PSO method is run 300 times (30 runs x 10 folds) for each dataset. The average classification accuracies are reported and compared using Wilcoxon statistical significance test [39], with a 5\% significance level. Experiments were runs on PC with Intel Core i7-4770 CPU @ $3.4 \mathrm{GHz}$ and a total memory of $8 \mathrm{~GB}$.

\section{RESUlTS AND Discussions}

Table III shows the best and average test accuracy of KNN using the original feature set ("Full"), and the feature subset returned by the four PSO-based FS methods on each dataset. The reported accuracy is the balanced accuracy calculated using Eq. (11). The third and fourth columns show the running time (in minutes) and feature subset sizes. The smallest running time, size and the highest average accuracy obtained on each dataset are bold. Columns $S_{1}$ and $S_{2}$ display the Wilcoxon significance test results (with a significance level of 0.05 ) of the corresponding method over VLPSO and VLPSOLS, respectively. "+" or "-" means the result is significantly better or worse than the proposed method and "=" means they are similar in the Wilcoxon tests. In other words, the more "-", the better the proposed methods.
TABLE III AVERAGE TEST RESULTS.

\begin{tabular}{|c|c|c|c|c|c|c|c|}
\hline Dataset & Method & Time(m) & Size & Best & Mean \pm Std & $S_{1}$ & $S_{2}$ \\
\hline \multirow{6}{*}{ SRBCT } & Full & & 2308.0 & 87.08 & & - & - \\
\hline & PSO & 8.2 & 1119.4 & 92.50 & $89.51 \pm 1.56$ & - & - \\
\hline & ECLPSO & 7.5 & 1054.8 & 90.42 & $88.10 \pm 1.57$ & - & - \\
\hline & $\mathrm{CSO}$ & 19.9 & 85.4 & 100.00 & $93.29 \pm 3.52$ & - & - \\
\hline & VLPSO & 1.4 & 49.1 & 100.00 & $99.67 \pm 0.52$ & & $=$ \\
\hline & VLPSO-LS & 2.1 & 71.4 & 100.00 & $99.75 \pm 0.45$ & & \\
\hline \multirow{6}{*}{ DLBCL } & Full & & 5469.0 & 83.00 & & - & $\begin{array}{ll}- \\
-\end{array}$ \\
\hline & PSO & 47.6 & 2681.0 & 86.33 & $83.67 \pm 1.52$ & - & - \\
\hline & ECLPSO & 44.2 & 2491.3 & 86.33 & $82.44 \pm 2.01$ & - & - \\
\hline & $\mathrm{CSO}$ & 394.8 & 30.1 & 100.00 & $94.30 \pm 4.05$ & + & $=$ \\
\hline & VLPSO & 7.4 & 48.1 & 93.33 & $86.51 \pm 2.88$ & & - \\
\hline & VLPSO-LS & 8.8 & 59.9 & 99.17 & $96.13 \pm 1.90$ & & \\
\hline \multirow{6}{*}{ 9Tumor } & Full & & 5726.0 & 36.67 & & - & - \\
\hline & PSO & 39.2 & 2811.9 & 45.00 & $42.72 \pm 1.42$ & - & - \\
\hline & ECLPSO & 39.2 & 2605.5 & 45.00 & $41.33 \pm 1.48$ & - & - \\
\hline & $\mathrm{CSO}$ & 373.4 & 220.3 & 68.33 & $\mathbf{5 9 . 5 0} \pm \mathbf{3 . 7 2}$ & + & + \\
\hline & VLPSO & 6.2 & 44.2 & 61.67 & $55.11 \pm 4.71$ & & $=$ \\
\hline & VLPSO-LS & 6.2 & 61.9 & 70.00 & $56.78 \pm 5.23$ & & \\
\hline \multirow{6}{*}{ Leuk1 } & Full & & 5327.0 & 79.72 & & - & - \\
\hline & PSO & 41.2 & 2615.5 & 87.36 & $80.60 \pm 2.55$ & - & - \\
\hline & ECLPSO & 36.3 & 2427.9 & 87.64 & $80.88 \pm 2.28$ & - & - \\
\hline & $\mathrm{CSO}$ & 251.8 & 170.1 & 96.81 & $88.45 \pm 3.90$ & - & - \\
\hline & VLPSO & 6.4 & 54.7 & 97.92 & $93.31 \pm 2.34$ & & $=$ \\
\hline & VLPSO-LS & 7.9 & 59.3 & 95.42 & $93.75 \pm 1.56$ & & \\
\hline \multirow{6}{*}{ Brain1 } & Full & & 5920.0 & 72.08 & & $=$ & - \\
\hline & PSO & 66.7 & 2917.2 & 77.08 & $73.73 \pm 2.21$ & + & - \\
\hline & ECLPSO & 60.0 & 2710.0 & 77.08 & $73.87 \pm 2.37$ & + & - \\
\hline & $\mathrm{CSO}$ & 462.1 & 207.6 & 86.67 & $79.93 \pm 3.09$ & + & + \\
\hline & VLPSO & 9.8 & 26.8 & 79.17 & $71.19 \pm 3.52$ & & - \\
\hline & VLPSO-LS & 13.0 & 102.1 & 81.25 & $75.54 \pm 2.79$ & & \\
\hline \multirow{6}{*}{ Leuk2 } & Full & & 11225.0 & 89.44 & & - & - \\
\hline & PSO & 120.6 & 5535.7 & 92.22 & $89.83 \pm 1.00$ & - & - \\
\hline & ECLPSO & 125.6 & 5115.6 & 92.22 & $89.82 \pm 1.20$ & - & - \\
\hline & $\mathrm{CSO}$ & 1845.2 & 88.6 & 98.33 & $91.72 \pm 3.16$ & $=$ & - \\
\hline & VLPSO & 16.9 & 35.2 & 94.44 & $91.56 \pm 1.67$ & & - \\
\hline & VLPSO-LS & 18.3 & 61.2 & 96.67 & $\mathbf{9 5 . 3 9} \pm \mathbf{0 . 9 5}$ & & \\
\hline \multirow{6}{*}{ Brain2 } & Full & & 10367.0 & 62.50 & & - & - \\
\hline & PSO & 80.5 & 5117.2 & 67.08 & $61.99 \pm 2.91$ & - & - \\
\hline & ECLPSO & 73.6 & 4718.7 & 68.75 & $63.20 \pm 2.60$ & - & - \\
\hline & $\mathrm{CSO}$ & 950.8 & 90.43 & 90.83 & $80.44 \pm 6.28$ & + & + \\
\hline & VLPSO & 12.1 & 81.5 & 73.33 & $66.78 \pm 4.10$ & & - \\
\hline & VLPSO-LS & 13.7 & 61.4 & 82.92 & $73.25 \pm 4.30$ & & \\
\hline \multirow{6}{*}{ Prostate } & Full & & 10509.0 & 85.33 & & - & - \\
\hline & PSO & 160.6 & 5193.7 & 88.33 & $86.00 \pm 1.49$ & - & - \\
\hline & ECLPSO & 152.5 & 4818.5 & 88.33 & $85.46 \pm 1.41$ & - & - \\
\hline & $\mathrm{CSO}$ & 2369.9 & 357.2 & 95.17 & $88.99 \pm 2.68$ & $=$ & - \\
\hline & VLPSO & 22.6 & 26.4 & 94.17 & $89.82 \pm 2.28$ & & - \\
\hline & VLPSO-LS & 25.8 & 56.4 & 97.17 & $92.58 \pm 1.47$ & & \\
\hline \multirow{6}{*}{ Lung } & Full & & 12600.0 & 78.05 & & - & - \\
\hline & PSO & 574.2 & 6234.7 & 82.72 & $78.77 \pm 1.53$ & - & - \\
\hline & ECLPSO & 503.1 & 5739.7 & 81.64 & $77.91 \pm 1.98$ & - & - \\
\hline & $\mathrm{CSO}$ & 5565.9 & 226.4 & 93.79 & $87.72 \pm 2.93$ & - & - \\
\hline & VLPSO & 70.1 & 176.1 & 94.08 & $89.47 \pm 2.18$ & & $=$ \\
\hline & VLPSO-LS & 307.1 & 242.9 & 93.71 & $90.17 \pm 2.10$ & & \\
\hline \multirow{6}{*}{ 11Tumor } & Full & & 12533.0 & 71.42 & & - & - \\
\hline & PSO & 418.5 & 6205.0 & 75.59 & $71.81 \pm 1.75$ & - & - \\
\hline & ECLPSO & 366.7 & 5731.7 & 74.09 & $71.09 \pm 1.20$ & - & - \\
\hline & $\mathrm{CSO}$ & 6288.6 & 588.6 & 84.47 & $79.52 \pm 2.35$ & $=$ & - \\
\hline & VLPSO & 65.8 & 246.7 & 85.16 & $80.81 \pm 2.32$ & & - \\
\hline & VLPSO-LS & 99.0 & 367.4 & 86.51 & $82.81 \pm 2.09$ & & \\
\hline
\end{tabular}




\section{A. VLPSO Results}

1) VLPSO versus Full: As can be seen from Table III, the numbers of features selected by VLPSO on all datasets were one to two orders of magnitude smaller than the original size with the best ratio of 1/398 in Prostate. Among all the compared methods, VLPSO obtained the smallest feature subsets on almost all datasets. With the smallest size, feature subsets returned by VLPSO significantly improved the performance of KNN on eight out of ten datasets. The highest improvement was seen on 9Tumor with $18.4 \%$ increase on average and $25 \%$ in the best case. On SRBCT, the proposed method selected less than 50 features to achieve $100 \%$ accuracy in almost all 300 runs, which is more than $12 \%$ improvement on Full. On Brain1, VLPSO selected about 27 out of 5920 features to obtain a similar classification performance as Full on average and 7\% higher accuracy in the best case.

2) VLPSO versus Standard PSO: Although PSO reduced the original feature sets by half, VLPSO still selected at least an order of magnitude fewer features than PSO on all datasets and achieved significantly better performance than PSO on nine datasets with the highest improvement of $12.7 \%$ on Leuk1. The highest dimensionality reduction was seen in Prostate where VLPSO selected 197 times fewer features than PSO and still improved $3.8 \%$ on the average PSO performance. Only on Brain1, VLPSO obtained $2.5 \%$ lower average accuracy than PSO while selected 109 times fewer features. However, the best accuracy achieved by VLPSO on Brain1 is still 2\% higher than PSO.

3) VLPSO versus ECLPSO: Although ECLPSO selected a smaller number of features than PSO on all datasets, its performance was quite similar to PSO with a maximum $2 \%$ difference in accuracy. Compared with ECLPSO, VLPSO also selected a much smaller number of features and achieved significantly better performance on all datasets except for Brain1.

4) VLPSO versus CSO: Compared with VLPSO, CSO selected more features on nine datasets. On Prostate, CSO selected 357 features while VLPSO selected 26 features only. In terms of classification accuracy, VLPSO achieved a significantly better or similar classification performance as CSO on six datasets, and worse on the remaining four datasets.

In summary, VLPSO won 32, draw 4 and lost 4 out of the 40 comparisons in terms of classification performance while selecting the smallest feature subsets in almost all cases. Its results indicated that VLPSO conducted a much better search than the compared methods. VLPSO effectiveness is contributed by two mechanisms, the population division and length changing, which are enabled by using the variablelength representation to encode candidate solutions with different lengths. The population division distributes particles in the swarm into different areas of the search space, which effectively ensures the diversity of the swarm. Furthermore, when there is a sign of being stuck in local optima, the proposed length changing mechanism enabled particles to change their search space without throwing away the knowledge that they have learnt so far. This mechanism also gradually adjusts PSO search to focus on smaller and more fruitful areas, enabling PSO to find much smaller feature subsets with better discriminating ability.

\section{B. VLPSO-LS Results}

1) VLPSO-LS versus Full: As can be seen from Table III, the number of features selected by VLPSO-LS on all datasets was one to two orders of magnitude smaller than the original size. The features selected by VLPSO-LS helped KNN obtain significantly better accuracy than using Full on all datasets with an increase of more than $10 \%$ on seven datasets. On 9Tumor, VLPSO-LS subsets obtained 20\% higher accuracy than Full on average and 33\% higher in the best case.

2) VLPSO-LS versus Standard PSO and ECLPSO: The results of significance test shown in Column $S_{2}$ showed that VLPSO-LS outperformed PSO on all datasets while selected 16 to 92 times smaller number of features. Seven out of ten datasets witnessed an increase of at least $10 \%$ on average accuracy with the highest improvement of $14 \%$ on 9Tumor.

3) VLPSO-LS versus ECLPSO: Comparison between ECLPSO and VLPSO-LS yields a similar pattern as with PSO where VLPSO-LS selected 14 to 85 times smaller number of features than ECLPSO to achieve significantly better performance on all datasets.

4) VLPSO-LS versus CSO: Although CSO selected a much smaller number of features than PSO and ECLPSO, its feature subsets were still up to 6.3 times larger than VLPSO-LS on eight datasets. VLPSO obtained significantly better classification performance than CSO on six datasets that had up to $6.4 \%$ higher average accuracy, similar on one and worse on the remaining three datasets, namely 9Tumor, Brain1 and Brain2.

5) VLPSO-LS versus VLPSO: As shown in Table III, VLPSO-LS selected slightly more features than VLPSO on almost all cases to further improve the performance of VLPSO on six datasets. The highest improvement was on DLBCL with $9.6 \%$ higher accuracy. On Leuk2, VLPSO-LS selected 20 features less than VLPSO while increased VLPSO accuracy by $6.5 \%$ on average and $9.6 \%$ in the best case. While VLPSO obtained a similar or worse performance than Full and other PSO methods on Brain1, VLPSO-LS significantly outperformed the others on this dataset.

In summary, VLPSO-LS won 42, draw 5 and lost 3 out of 50 comparisons. The results of VLPSO-LS indicated that by removing redundant features and adding more relevant ones, the local search strategy helped VLPSO fine tune its solutions to achieve the highest accuracy on all the datasets.

\section{Computation Time}

As can be seen from the third column of Table III, the fastest algorithm among all the five compared methods is VLPSO. Although VLPSO-LS performed more fitness evaluations, it is the second-fastest method with only a slightly longer time than VLPSO. PSO and ECLPSO, in third place, are similar with their running time 5 to 8 times longer than VLPSO on all datasets. Finally, CSO required the longest running time at 14 to 109 times longer than VLPSO. This may be due to the strategy of recording the historical fitness values of all 

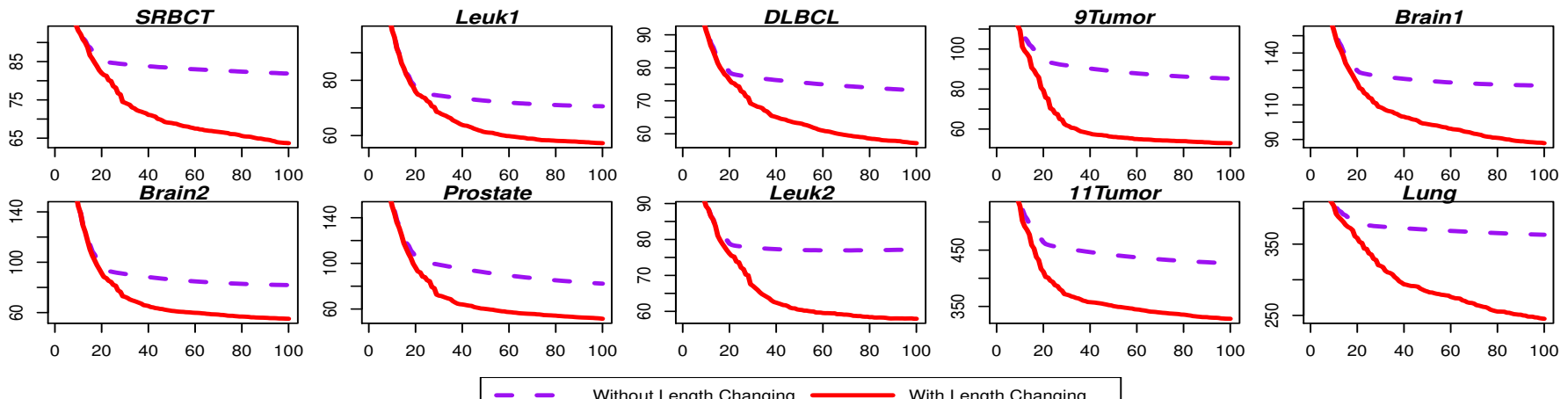

Fig. 6. The average feature subset size of the whole swarm from iteration 10 to 100 in both scenarios with and without using length changing.

previously selected feature subset in an archive to avoid reevaluating the same solution. This strategy has been shown to be effective in [21] where the largest dataset had 1588 features. However, when the number of features increases to tens of thousands of features, the evaluation time saved seemed to be affected by the time needed for matching the archived solutions which used a fix-length representation with its length equal to the original number of features.

In summary, the variable-length PSO-based methods required a much shorter running time than the traditional fixlength ones. Section VI will further investigates the differences in evolutionary processes that contribute to the effectiveness and efficiency of the proposed methods.

\section{The Effect of Length Changing}

To investigate the effect of length changing, we analyse the results of VLPSO-LS with (W) and without (WO) applying length changing mechanism, which is shown in Table $\mathrm{V}$ given in the Appendix. Compared to WO, W obtained up to 10 fewer features on all datasets except for Lung where it selected 1.8 more features on average. In terms of the classification accuracy, $\mathrm{W}$ results were significantly better than WO on DLBCL and similar on the remaining datasets. Furthermore, $\mathrm{W}$ saved up to $14 \%$ of WO running time on all datasets. Fig. 6 shows the average feature subset size of the whole swarm from iteration 10 (i.e. just after the first time length changing is applied in the evolutionary process) to 100 in both scenarios. The figure shows that length changing dramatically reduced particle lengths, which required a shorter time for PSO updating.

\section{E. Comparisons with Traditional Methods}

To see if the proposed methods performed better than the traditional FS methods, we compared the results of VLPSOLS with those returned from LFS, CFS and FCBF, all of which can automatically determine the number of features that should be selected. Table IV showed the running time, the returned feature subset size, and the best and mean accuracy of each method. Column $S$ showed the results of Wilcoxon significance test compared the corresponding method over VLPSO-LS using the same symbols and meanings as in Table III. The smallest size, the highest average and best accuracy obtained on each dataset are bold.
TABLE IV

VLPSO-LS VERSUS TRADITIONAL METHODS.

\begin{tabular}{|c|c|c|c|c|c|c|}
\hline Dataset & Method & Time (s) & Size & Best & Mean \pm Std & $S$ \\
\hline \multirow{4}{*}{ SRBCT } & LFS & 25.0 & 7.1 & 91.67 & \multirow[b]{4}{*}{$99.75 \pm \mathbf{0 . 4 5}$} & - \\
\hline & CFS & 243.3 & 112.3 & 99.17 & & - \\
\hline & FCBF & 1.4 & 69.0 & 98.75 & & - \\
\hline & VLPSO-LS & 123.3 & 71.4 & 100.00 & & \\
\hline \multirow{4}{*}{ DLBCL } & LFS & 56.3 & 5.9 & 83.33 & \multirow[b]{4}{*}{$96.13 \pm 1.90$} & - \\
\hline & CFS & 778.4 & 86.3 & 93.00 & & - \\
\hline & FCBF & 1.6 & 66.1 & 94.83 & & - \\
\hline & VLPSO-LS & 527.3 & 59.9 & 99.17 & & \\
\hline \multirow{4}{*}{ 9Tumor } & LFS & 52.9 & 9.7 & 26.67 & \multirow[b]{4}{*}{$56.78 \pm 5.23$} & - \\
\hline & CFS & 341.2 & 44.0 & 56.67 & & $=$ \\
\hline & FCBF & 1.7 & 33.7 & 55.00 & & \\
\hline & VLPSO-LS & 371.4 & 61.9 & 70.00 & & \\
\hline \multirow{4}{*}{ Leuk1 } & LFS & 51.9 & 5.4 & 85.14 & \multirow[b]{4}{*}{$93.75 \pm 1.56$} & - \\
\hline & CFS & 778.4 & 79.4 & 92.08 & & - \\
\hline & FCBF & 1.4 & 48.5 & 89.86 & & - \\
\hline & VLPSO-LS & 471.5 & 59.3 & 95.42 & & \\
\hline \multirow{4}{*}{ Brain1 } & LFS & 77.9 & 12.2 & 63.33 & \multirow[b]{4}{*}{$75.54 \pm 2.79$} & - \\
\hline & CFS & 2973.0 & 151.9 & 76.67 & & + \\
\hline & FCBF & 2.8 & 104.6 & 73.75 & & \\
\hline & VLPSO-LS & 781.6 & 102.1 & 81.25 & & \\
\hline \multirow{4}{*}{ Leuk2 } & LFS & 143.4 & 4.7 & 89.44 & \multirow[b]{4}{*}{$95.39 \pm 0.95$} & - \\
\hline & CFS & 5653.0 & 129.5 & 94.44 & & - \\
\hline & FCBF & 4.1 & 77.5 & 95.56 & & $=$ \\
\hline & VLPSO-LS & 1099.1 & 61.2 & 96.67 & & \\
\hline \multirow{4}{*}{ Brain2 } & LFS & 113.9 & 9.1 & 77.50 & \multirow[b]{4}{*}{$73.25 \pm 4.30$} & + \\
\hline & CFS & 3182.2 & 101.1 & 77.50 & & + \\
\hline & FCBF & 2.7 & 66.2 & 77.50 & & + \\
\hline & VLPSO-LS & 820.6 & 61.4 & 82.92 & & \\
\hline \multirow{4}{*}{ Prostate } & LFS & 158.2 & 5.9 & 90.17 & \multirow[b]{4}{*}{$92.58 \pm 1.47$} & - \\
\hline & CFS & 2537.4 & 80.4 & 92.17 & & $=$ \\
\hline & FCBF & 3.4 & 66.1 & 92.17 & & $=$ \\
\hline & VLPSO-LS & 1550.5 & 56.4 & 97.17 & & \\
\hline \multirow{4}{*}{ Lung } & LFS & 358.8 & 8.5 & 79.62 & \multirow[b]{4}{*}{$90.17 \pm 2.10$} & - \\
\hline & CFS & 85179.1 & 517.0 & 93.76 & & + \\
\hline & FCBF & 56.7 & 439.4 & 92.71 & & + \\
\hline & VLPSO-LS & 18425.0 & 242.9 & 93.71 & & \\
\hline \multirow{4}{*}{ 11Tumor } & LFS & 309.3 & 17.3 & 61.76 & \multirow[b]{4}{*}{$82.81 \pm \mathbf{2 . 0 9}$} & - \\
\hline & CFS & 57340.7 & 361.6 & 80.04 & & - \\
\hline & FCBF & 31.1 & 349.6 & 80.57 & & - \\
\hline & VLPSO-LS & 5941.5 & 367.4 & 86.51 & & \\
\hline
\end{tabular}

1) VLPSO-LS versus LFS: As can be seen from Table IV, LFS selected less than 20 features on all datasets, obtaining the smallest feature subset of all the compared methods. However, these smallest subsets obtained significantly lower accuracy than VLPSO-LS on nine datasets with more than $10 \%$ difference on five cases. On 9Tumor, VLPSO-LS selected 52 more features to achieve 30\% higher accuracy than LFS on average and $43 \%$ higher in the best case. Only on Brain2, VLPSO-LS obtained a lower average accuracy than LFS, but in the best case, VLPSO-LS still achieved more than 5\% higher accuracy than LFS. The results indicate that the linear forward search in LFS was trapped in local optima in a very 
early stage, resulting in minimal but low performance feature sets.

2) VLPSO-LS versus CFS: Compared with CFS, VLPSOLS selected fewer features on eight datasets to obtain significantly better accuracies on five, and similar on two. Although both had a similar classification performance on 9Tumor, VLPSO-LS still obtained 14\% higher accuracy in the best case. On Brain1 and Lung, VLPSO-LS had 1-3\% lower average accuracy; however, with much smaller feature subsets and could achieve better accuracy in the best case. We also note that although CFS is a deterministic and filter FS method, its running time is two times longer than VLPSO-LS on the small SRBCT dataset, and nine times on the large 11Tumor dataset. This indicates that VLPSO-LS better scale to high-dimensional data than CFS.

3) VLPSO-LS versus FCBF: The fourth column of Table IV showed that the difference in feature subset size between VLPSO-LS and FCBF was very small on all datasets except Lung, where VLPSO-LS selected about a half of FCBF. With a similar size, feature subsets of VLPSO-LS obtained a significantly better accuracy than FCBF on five datasets, and similar on two. Selecting 11 more features on Leuk1, VLPSOLS achieved $3.9 \%$ higher accuracy than FCBF on average and $5.6 \%$ higher in the best case. On Brain2, although VLPSO-LS had $4.3 \%$ lower average accuracy, its best accuracy is still $5.4 \$$ higher than FCBF. We also note that FCBF scaled very well to high-dimensional data and it is the fastest method among the four, which is an advantage of a filter and ranking FS method. However, the inferior results of FCBF suggested that the heuristic search of FCBF in the second stage might get stuck in local optima while the global search helped PSO overcome this problem to obtain better results.

In summary, among 30 comparisons with the three methods, VLPSO-LS won 19 cases, drew 6 and lost 5. The results showed that VLPSO-LS achieved a significantly better performance than the traditional methods in reasonable running time.

\section{FurTher ANALYSIS}

We have shown so far that in most cases, VLPSO and VLPSO-LS achieved much better performance than the compared FS methods in terms of classification accuracy, dimensionality reduction and computation time. In this section, we will further investigate their performance to reveal the contributions of different components to improving PSO search capability. Specifically, we will investigate the effect of variable-length representation and the local search. Note that the results shown in all figures of this section are averaged over the 30 runs.

\section{A. Efficiency of Variable-Length PSO Representation}

First of all, we will investigate the effect of variable-length representation on the tremendous reduction of computation time. Since all the four PSO-based FS methods used the same population size, the maximum number of iterations, and the fitness function, their running time difference is contributed by the feature subset size which affected the fitness evaluation time and the length of particles which affected the particle updating time. To investigate these differences, we plot the average feature subset size of a particle in each iteration of all the four methods and the particles' maximum length of the two proposed methods in Figs. 7 and 8, respectively.

As can be seen from Fig. 7, the subset sizes of PSO and ECLPSO particles started quite large and slightly increased over the whole evolutionary process. On the other hand, starting at about half size of PSO, VLPSO witnessed a steady decrease in the first 40 iterations and then a slight decline in the remaining stage. Starting at the same point as VLPSO, VLPSO-LS dramatically dropped to a very small size in the first several iterations and kept stable till the end. These figures clearly showed that the fitness evaluation time in VLPSO and VLPSO-LS are significantly reduced thanks to the small subset sizes of all particles.

In addition to the small evaluation time, the particle updating time of the variable-length PSO is also much smaller. With a fix-length representation, PSO and ECLPSO spent a fixed amount of time to update particles with the length of the original number of features in every iteration. On the other hand, using the size division strategy, VLPSO and VLSPOLS spent much shorter time to update their particles from the first iteration to the end. Furthermore, after each length changing, the particle lengths even get significantly shorter. Fig. 8 showed the maximum length of particles in VLPSO and VLSPO-LS changing during the evolutionary process of all datasets. The figures showed that the maximum length dramatically dropped in the first 40 iterations and slightly decreased after that. The significant impact of particle lengths on the running time can be shown in the Lung dataset. In this dataset, while the average feature subset sizes of VLPSO-LS were much smaller than VLPSO in the first 40 iterations as shown in Fig. 7, the particles' maximum length in VLPSO-LS is much larger than VLPSO as shown in Fig. 8, which makes VLPSO-LS had a much longer running time than VLPSO as shown in Table III.

\section{B. Effectiveness of Variable-Length PSO Representation}

Secondly, we will investigate the effect of variable-length representation on the size and accuracy of the returned feature subsets. Fig. 9 and 10 show the changing of gbest size and fitness during the evolutionary process. Note that the fitness values are calculated based on Eq. (10), which is a combination of KNN accuracy and the distance measure. Therefore, these values hardly reached the value of 1 .

As shown in Fig. 9, from the beginning of the run, gbest size of the two variable-length PSO methods was already way smaller than the fixed ones. By dividing particles into different length divisions, the swarm in the proposed methods could have a higher diversity than the baseline methods. This enabled them to find much smaller feature subsets from the early stage of the evolutionary process. By removing redundant features from pbest, VLPSO-LS had an even smaller gbest size than VLPSO from the beginning and kept nearly unchanged to the end. On the other hand, VLSPO's gbest maintained a gradual decrease over the whole evolutionary process and reached a smaller subset size than VLPSO-LS at the end. 

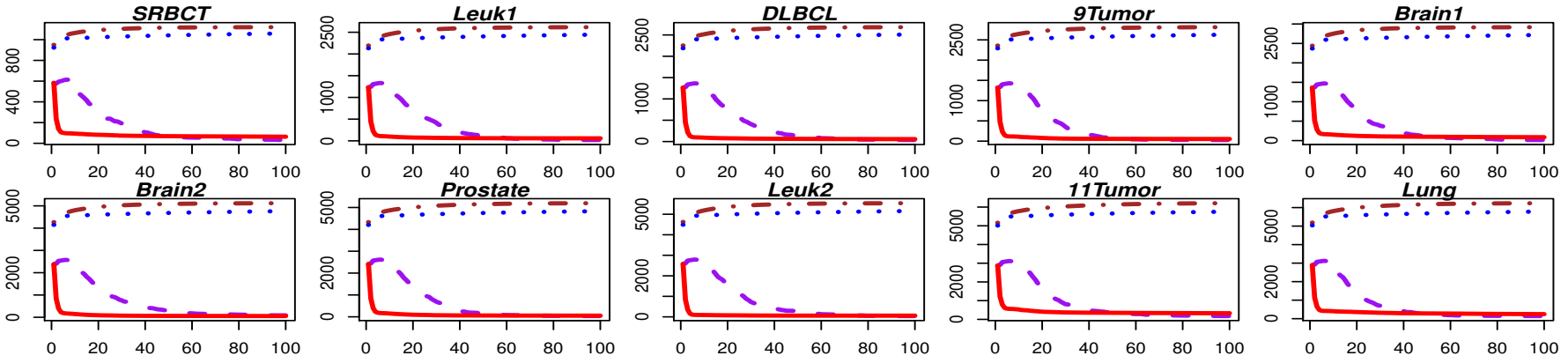

. - . . PSO ...... ECLPSO $\quad-\quad-$ VLPSO

Fig. 7. The average feature subset size of the whole swarm in 100 iterations.
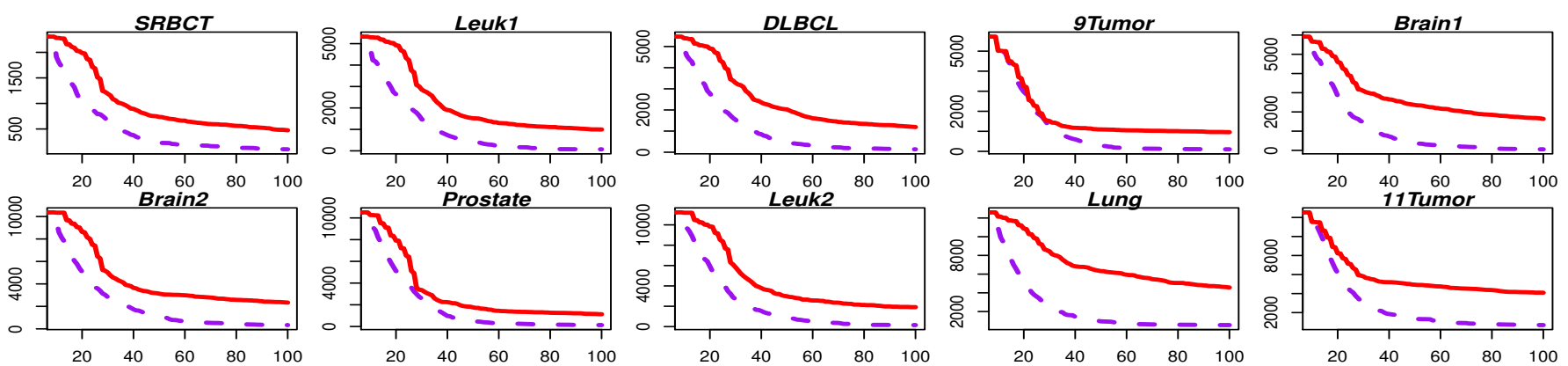

- - V VLPSO VLPSO-LS

Fig. 8. The average particle maximum length in 100 iterations.
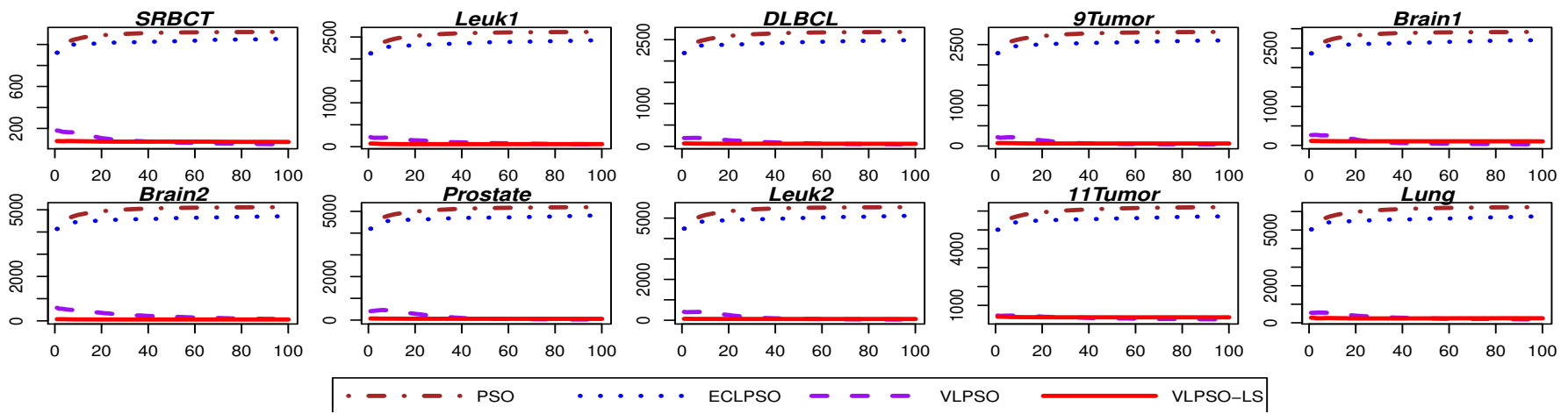

Fig. 9. The average feature subset size of gbest in 100 iterations.

As shown in Fig. 10, with a small subset size, VLPSO's gbest obtained a much higher fitness than the baseline methods from the first iteration and continued to improve to the end. Using an informative local search to remove redundant features and add more relevant ones, VLPSO-LS's gbest even had much better fitness than VLPSO. The gap between gbest's fitness of both methods varied in different datasets; however, with the same trend which is getting closer at the end. The gap's magnitude may reflect the complexity of the corresponding search space. For example in SRBCT, a small dataset with 2038 features, this gap is quite small, and the significance test on 30 runs showed that both methods obtained a similar classification accuracy while VLPSO-LS selected 20 more features than VLPSO. On the other hand, on such datasets with a greater number of classes and features as 9Tumor and 11Tumor, the gap is quite large even at the end.

\section{CONCLUSIONS}

This study aims to propose a new PSO representation that can have a variable and dynamic length for FS on highdimensional data. The goal was achieved by proposing a new variable-length PSO-based FS method where particles in a swarm can have different lengths which can also be changed during the evolutionary process. The results showed that the proposed variable-length PSO-based methods achieved a much smaller feature subset with better classification performance in a shorter time than the traditional fixed-length methods. By having shorter and dynamic lengths to encode particles, PSO maintains a better diversity in the swarm and requires a much smaller number of updating operations. The proposed length changing mechanism also helped PSO jump out of local optima and focus its search on a more fruitful area. 

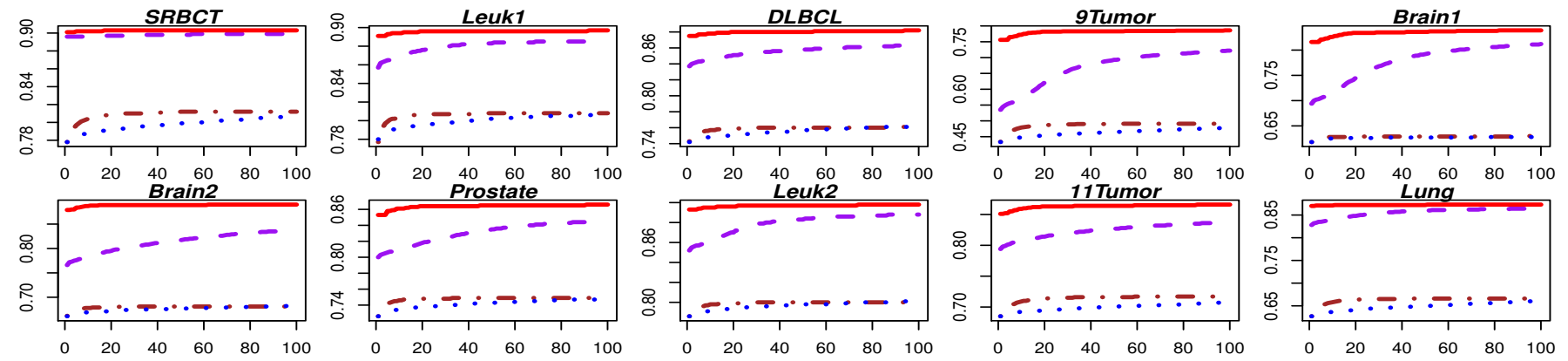

Fig. 10. The average fitness of gbest in 100 iterations.

The proposed variable-length PSO-based method has shown promise in FS. It can also be applied to other tasks. In the proposed representation, each dimension is updated separately without taking into account other dimensions. This may limit the performance of PSO in FS on problems which have strong interactions between features for target prediction. Taking this information into account when selecting features may help PSO obtain even better results, but it is very hard to achieve. This direction will be considered in our future work.

\section{ACKNOWLEDGMENT}

This work was supported in part by the Marsden Fund of New Zealand Government under Contracts VUW1509 and VUW1615, Huawei Industry Fund E2880/3663, and the University Research Fund at Victoria University of Wellington 209862/3580, and 213150/3662.

\section{APPENDIX}

TABLE V

COMPARED RESULTS OF VLPSO-LS IN BOTH SCENARIOS WITH (W) AND WITHOUT (WO) USING THE LENGTH CHANGING MECHANISM

\begin{tabular}{|l|l|r|r|r|r|r|}
\hline Dataset & Method & Time $(\mathbf{m})$ & Size & Best & Mean \pm Std & $S$ \\
\hline \multirow{2}{*}{ SRBCT } & WO & 2.10 & 76.3 & 100.00 & $99.89 \pm 0.29$ & $=$ \\
& W & 2.05 & 71.4 & 100.00 & $99.75 \pm 0.45$ & \\
\hline \multirow{2}{*}{ DLBCL } & WO & 9.14 & 62.5 & 98.33 & $95.03 \pm 2.00$ & - \\
& W & 8.79 & 59.9 & 99.17 & $96.13 \pm 1.90$ & \\
\hline \multirow{2}{*}{ TTumor } & WO & 7.19 & 67.6 & 63.33 & $55.89 \pm 3.93$ & $=$ \\
& W & 6.19 & 61.9 & 70.00 & $56.78 \pm 5.23$ & \\
\hline \multirow{2}{*}{ Leuk1 } & WO & 8.74 & 61.8 & 97.64 & $93.63 \pm 2.09$ & $=$ \\
& W & 7.86 & 59.3 & 95.42 & $93.75 \pm 1.56$ & \\
\hline \multirow{2}{*}{ Brain1 } & WO & 13.39 & 107.6 & 82.08 & $76.15 \pm 3.41$ & $=$ \\
& W & 13.03 & 102.1 & 81.25 & $75.54 \pm 2.79$ & \\
\hline \multirow{2}{*}{ Leuk2 } & WO & 20.64 & 65.4 & 98.33 & $95.26 \pm 1.37$ & $=$ \\
& W & 18.32 & 61.2 & 96.67 & $95.39 \pm 0.95$ & \\
\hline \multirow{2}{*}{ Brain2 } & WO & 14.99 & 67.0 & 79.58 & $73.50 \pm 3.55$ & $=$ \\
& W & 13.68 & 61.4 & 82.92 & $73.25 \pm 4.30$ & \\
\hline \multirow{2}{*}{ Prostate } & WO & 27.14 & 61.0 & 96.17 & $92.49 \pm 1.93$ & $=$ \\
& W & 25.84 & 56.4 & 97.17 & $92.58 \pm 1.47$ & \\
\hline \multirow{2}{*}{ Lung } & WO & 312.96 & 241.1 & 94.19 & $90.14 \pm 2.14$ & $=$ \\
& W & 307.08 & 242.9 & 93.71 & $90.17 \pm 2.10$ & \\
\hline \multirow{2}{*}{11 Tumor } & WO & 102.09 & 377.3 & 85.96 & $82.79 \pm 1.84$ & $=$ \\
& W & 99.02 & 367.4 & 86.51 & $82.81 \pm 2.09$ & \\
\hline
\end{tabular}

\section{REFERENCES}

[1] M. Dash, "Feature selection via set cover," in Proceedings of IEEE Knowledge and Data Engineering Exchange Workshop, Nov 1997, pp. $165-171$.

[2] I. Guyon and A. Elisseeff, "An introduction to variable and feature selection," Journal of Machine Learning Research, vol. 3, pp. 11571182, 2003.
[3] B. Tran, B. Xue, and M. Zhang, "A PSO based hybrid feature selection algorithm for high-dimensional classification," in Proceedings of IEEE Congress on Evolutionary Computation, 2016, pp. 3801-3808.

[4] Y. Sun, IEEE Transactions on Pattern Analysis and Machine Isntelligence, vol. 29, no. 6, pp. 1035-1051, 2007.

[5] A. Jakulin and I. Bratko, "Testing the significance of attribute interactions," in Proceedings of the 21st International Conference on Machine Learning (ICML). ACM, 2004, pp. 52-59.

[6] L. Yu and H. Liu, "Feature selection for high-dimensional data: A fast correlation-based filter solution," in Proceedings of the 20th on International Conference on Machine Learning (ICML), 2003, pp. 856863.

[7] A. Whitney, "A direct method of nonparametric measurement selection," IEEE Transactions on Computers, vol. C-20, no. 9, pp. 1100-1103, 1971.

[8] T. Marill and D. M. Green, "On the effectiveness of receptors in recognition systems." IEEE Transactions on Information Theory, vol. 9 , no. 1 , pp. 11-17, 1963.

[9] R. Eberhart and J. Kennedy, "A new optimizer using particle swarm theory," in Proceedings of the 6th International Symposium on Micro Machine and Human Science, 1995, pp. 39-43.

[10] C. Sun, Y. Jin, R. Cheng, J. Ding, and J. Zeng, "Surrogate-assisted cooperative swarm optimization of high-dimensional expensive problems," IEEE Transactions on Evolutionary Computation, vol. 21, no. 4, pp. 644-660, 2017.

[11] C. Yue, B. Qu, and J. Liang, "A multi-objective particle swarm optimizer using ring topology for solving multimodal multi-objective problems," IEEE Transactions on Evolutionary Computation, vol. PP, no. 99, pp. 1-13, 2017. [Online]. Available: doi:10.1109/TEVC.2017.2754271

[12] B. Xue, M. Zhang, W. N. Browne, and X. Yao, "A survey on evolutionary computation approaches to feature selection," IEEE Transactions on Evolutionary Computation, vol. 20, no. 4, pp. 606-626, 2016.

[13] X. Li and X. Yao, "Cooperatively coevolving particle swarms for large scale optimization," IEEE Transactions on Evolutionary Computation, vol. 16, no. 2, pp. 210-224, 2012.

[14] J. J. Liang, A. K. Qin, P. N. Suganthan, and S. Baskar, "Comprehensive learning particle swarm optimizer for global optimization of multimodal functions," IEEE Transactions on Evolutionary Computation, vol. 10, no. 3, pp. 281-295, June 2006.

[15] X. Yu, Y. Liu, X. Feng, and G. Chen, "Enhanced comprehensive learning particle swarm optimization with exemplar evolution," in Proceedings of the 11th International Conference on Simulated Evolution and Learning (SEAL). Springer International Publishing, 2017, pp. 929-938.

[16] P. J. Angeline, Evolutionary optimization versus particle swarm optimization: Philosophy and performance differences. Springer Berlin Heidelberg, 1998, pp. 601-610.

[17] B. Xue, M. Zhang, and W. N. Browne, "Particle swarm optimisation for feature selection in classification: Novel initialisation and updating mechanisms," Applied Soft Computing, vol. 18, pp. 261-276, 2014.

[18] H. H. Inbarani, A. T. Azar, and G. Jothi, "Supervised hybrid feature selection based on pso and rough sets for medical diagnosis," Computer methods and programs in biomedicine, vol. 113, no. 1, pp. 175-185, 2014.

[19] B. Chakraborty and G. Chakraborty, "Fuzzy consistency measure with particle swarm optimization for feature selection," in IEEE International Conference on Systems, Man, and Cybernetics, 2013, pp. 4311-4315.

[20] R. Tahmasebifar, M. K. Sheikh-El-Eslami, and R. Kheirollahi, "Point and interval forecasting of real-time and day-ahead electricity prices by a 
novel hybrid approach," IET Generation, Transmission and Distribution, vol. 11, no. 9, pp. 2173-2183, 2017.

[21] S. Gu, R. Cheng, and Y. Jin, "Feature selection for high-dimensional classification using a competitive swarm optimizer," Soft Computing, vol. 22, no. 3, pp. 811-822, 2018.

[22] H. Banka and S. Dara, "A Hamming distance based binary particle swarm optimization (HDBPSO) algorithm for high dimensional feature selection, classification and validation," Pattern Recognition Letters, vol. 52, pp. 94-100, 2015.

[23] A. Moaref and V. S. Naeini, "A particle swarm optimization based on a ring topology for fuzzy-rough feature selection," in the 13th Iranian Conference on Fuzzy Systems (IFSC), 2013, pp. 1-6. [Online]. Available: doi:10.1109/IFSC.2013.6675598

[24] M. Lane, B. Xue, I. Liu, and M. Zhang, "Gaussian based particle swarm optimisation and statistical clustering for feature selection," in Evolutionary Computation in Combinatorial Optimisation (EvoCOP). Springer Berlin Heidelberg, 2014, vol. 8600, pp. 133-144.

[25] J. Kennedy and R. Eberhart, "A discrete binary version of the particle swarm algorithm," in IEEE International Conference on Systems, Man, and Cybernetics, vol. 5, 1997, pp. 4104-4108.

[26] B. H. Nguyen, B. Xue, and P. Andreae, "A novel binary particle swarm optimization algorithm and its applications on knapsack and feature selection problems," in The 20th Asia Pacific Symposium of Intelligent and Evolutionary Systems (IES). Springer International Publishing, 2017, pp. 319-332.

[27] W.-N. Chen, J. Zhang, H. S. Chung, W.-L. Zhong, W.-G. Wu, and Y.H. Shi, "A novel set-based particle swarm optimization method for discrete optimization problems," IEEE Transactions on Evolutionary Computation, vol. 14, no. 2, pp. 278-300, 2010.

[28] T. Hino, S. Ito, T. Liu, and M. Maeda, "Set-based particle swarm optimization with status memory for knapsack problem," Artificial Life and Robotics, vol. 21, no. 1, pp. 98-105, 2016.

[29] J. Langeveld and A. P. Engelbrecht, "Set-based particle swarm optimization applied to the multidimensional knapsack problem," Swarm Intelligence, vol. 6, no. 4, pp. 297-342, Dec 2012.

[30] W. H. Press, S. Teukolsky, W. Vetterling, and B. Flannery, "Numerical recipes in C," Cambridge University Press, vol. 1, p. 3, 1988.

[31] Q. Song, J. Ni, and G. Wang, "A fast clustering-based feature subset selection algorithm for high-dimensional data," IEEE Transactions on Knowledge and Data Engineering, vol. 25, pp. 1-14, 2013.

[32] H. Al-Sahaf, A. Al-Sahaf, B. Xue, M. Johnston, and M. Zhang, "Automatically evolving rotation-invariant texture image descriptors by genetic programming," IEEE Transactions on Evolutionary Computation, vol. 21, no. 1, pp. 83-101, 2017.

[33] G. Patterson and M. Zhang, "Fitness functions in genetic programming for classification with unbalanced data," in Proceedings of the 20th Australian Joint Conference on Artificial Intelligence (AI). Springer Berlin Heidelberg, 2007, pp. 769-775.

[34] C. C. Aggarwal, A. Hinneburg, and D. A. Keim, "On the surprising behavior of distance metrics in high dimensional space," in International Conference on Database Theory. Springer, 2001, pp. 420-434.

[35] M. A. Hall, "Correlation-based feature selection for discrete and numeric class machine learning," in Proceedings of the 7th International Conference on Machine Learning, 2000, pp. 359-366.

[36] M. Gutlein, E. Frank, M. Hall, and A. Karwath, "Large-scale attribute selection using wrappers," in IEEE Symposium on Computational Intelligence and Data Mining, 2009, pp. 332-339.

[37] M. Hall, E. Frank, G. Holmes, B. Pfahringer, P. Reutemann, and I. H Witten, "The weka data mining software: an update," ACM SIGKDD Explorations Newsletter, vol. 11, no. 1, pp. 10-18, 2009.

[38] G. Azevedo, G. Cavalcanti, and E. Filho, "An approach to feature selection for keystroke dynamics systems based on PSO and feature weighting," in IEEE Congress on Evolutionary Computation (CEC'07), 2007 , pp. 3577-3584.

[39] F. Wilcoxon, "Individual comparisons by ranking methods," Biometrics Bulletin, vol. 1, no. 6, pp. 80-83, 1945.

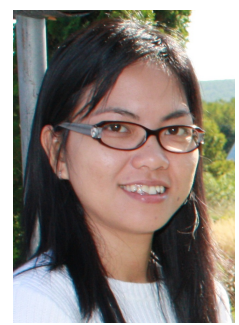

Binh Tran (S'14) received her B.E. in Computer Science from Cantho University, Vietnam, in 1998, the M.Sc. degree in Applied Computer Science from Free University of Brussels, Belgium, in 2002, and the $\mathrm{PhD}$ degree in computer science in 2018 at Victoria University of Wellington, New Zealand. She is currently a Post Doctoral Research Fellow in the School of Engineering and Computer Science at Victoria University of Wellington. Her research interests are in evolutionary computation, feature manipulation including feature selection and construction, high dimensional data, and machine learning.

Ms. Tran is a member of the IEEE Computational Intelligence Society (CIS). She has been serving as a reviewer for over 10 international journals and conferences in the field such as IEEE TEVC, IEEE TCYB, Applied Soft Computing, IEEE CEC, GECCO, SEAL, AAAI.

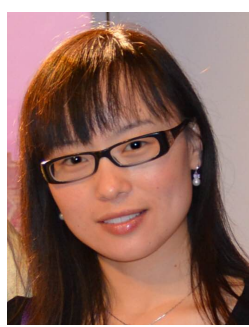

Bing Xue (M'10) received the B.Sc. degree from the Henan University of Economics and Law, Zhengzhou, China, in 2007, the M.Sc. degree in management from Shenzhen University, Shenzhen, China, in 2010, and the PhD degree in computer science in 2014 at Victoria University of Wellington, New Zealand. She is currently a Senior Lecturer in School of Engineering and Computer Science at Victoria University of Wellington. Her research focuses mainly on evolutionary computation, feature selection, feature construction, multi-objective optimisation, image analysis, transfer learning, data mining, and machine learning. She has over 100 papers published in fully refereed international journals and conferences and most of them are on evolutionary feature selection and construction.

Dr Xue is currently the Chair of the IEEE Task Force on Evolutionary Feature Selection and Construction, IEEE Computational Intelligence Society (CIS), Vice-Chair of the IEEE CIS Data Mining and Big Data Analytics Technical Committee, and Vice-Chair of IEEE CIS Task Force on Transfer Learning and Transfer Optimisation. She is also an Associate Editor/member of Editorial Board for five international journals and a reviewer of over 50 international journals. Dr Xue is the Finance Chair of IEEE Congress on Evolutionary Computation (CEC) 2019, a Program Co-Chair of the 31th Australasian AI 2018, ACALCI 2018, and the 7th International Conference on SoCPaR2015, and she is also a tutorial chair, special session chair, or publicity chair for many other international conferences.

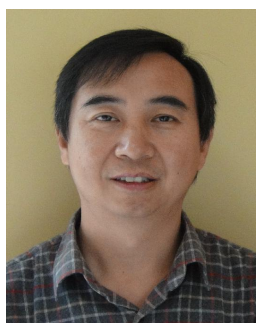

Mengjie Zhang (M'04-SM'10) received the B.E. and M.E. degrees from Artificial Intelligence Research Center, Agricultural University of Hebei, Hebei, China, and the Ph.D. degree in computer science from RMIT University, Melbourne, VIC, Australia, in 1989, 1992, and 2000, respectively. He is currently Professor of Computer Science, Head of the Evolutionary Computation Research Group, and the Associate Dean (Research and Innovation) in the Faculty of Engineering. His current research interests include evolutionary computation, particularly genetic programming, particle swarm optimization, and learning classifier systems with application areas of image analysis, multi-objective optimization, feature selection and reduction, job shop scheduling, and transfer learning. $\mathrm{He}$ has published over 350 research papers in refereed international journals and conferences.

Prof. Zhang is a Fellow of Royal Society of New Zealand and have been a Panel member of the Marsden Fund (New Zealand Government Funding). He is also a senior member of IEEE and a member of ACM. $\mathrm{He}$ is currently chairing the IEEE CIS Intelligent Systems and Applications Technical Committee, and the immediate Past Chair for the IEEE CIS Emergent Technologies Technical Committee and the Evolutionary Computation Technical Committee, and a member of the IEEE CIS Award Committee. $\mathrm{He}$ is a vice-chair of the IEEE CIS Task Force on Evolutionary Feature Selection and Construction, a vice-chair of the Task Force on Evolutionary Computer Vision and Image Processing, and the founding chair of the IEEE Computational Intelligence Chapter in New Zealand. He is also a committee member of the IEEE NZ Central Section. 